\title{
mGluR5 Modulation of Behavioral and Epileptic Phenotypes in a Mouse Model of Tuberous Sclerosis Complex
}

\author{
Elyza Kelly 1,2,9, Samantha M Schaeffer ${ }^{1,9}$, Sameer C Dhamne', Jonathan O Lipton ${ }^{1,3}$, Lothar Lindemann ${ }^{4}$, \\ Michael Honer ${ }^{5}$, Georg Jaeschke', Chloe E Super', Stephen HT Lammers', Meera E Modi', Jill L Silverman", \\ John R Dreier ${ }^{8}$, David J Kwiatkowski ${ }^{8}$, Alexander Rotenberg' and Mustafa Sahin*, \\ 'Department of Neurology, Boston Children's Hospital, F.M. Kirby Neurobiology Center, Harvard Medical School, Boston, MA, USA; ${ }^{2}$ Department \\ of Neuroscience, University of Texas Southwestern Medical Center at Dallas, Dallas, TX, USA; ${ }^{3}$ Division of Sleep Medicine, Harvard Medical School, \\ Boston, MA, USA; ${ }^{4}$ Roche Pharma Research and Early Development, Discovery Neuroscience, Neuroscience, Ophthalmology, and Rare Diseases, \\ Roche Innovation Center Basel, Basel, Switzerland; ${ }^{5}$ Roche Pharmaceuticals Research and Early Development, Translational Medicine \& \\ Biomarkers, Neuroscience, Ophthalmology, and Rare Diseases, Roche Innovation Center Basel, Basel, Switzerland; ${ }^{6}$ Roche Pharmaceuticals \\ Research and Early Development, Therapeutic Modalities, Small Molecule Research, Roche Innovation Center Basel, Basel, Switzerland; \\ ${ }^{7}$ Department of Psychiatry and Behavioral Sciences, MIND Institute, University of California Davis School of Medicine, Sacramento, CA, USA; \\ ${ }^{8}$ Department of Medicine, Brigham and Women's Hospital, Boston, Massachusetts, USA
}

\begin{abstract}
Drugs targeting metabotropic glutamate receptor 5 (mGluR5) have therapeutic potential in autism spectrum disorders (ASD), including tuberous sclerosis complex (TSC). The question whether inhibition or potentiation of mGluR5 could be beneficial depends, among other factors, on the specific indication. To facilitate the development of mGluR5 treatment strategies, we tested the therapeutic utility of mGluR5 negative and positive allosteric modulators (an mGluR5 NAM and PAM) for TSC, using a mutant mouse model with neuronal loss of Tsc2 that demonstrates disease-related phenotypes, including behavioral symptoms of ASD and epilepsy. This model uniquely enables the in vivo characterization and rescue of the electrographic seizures associated with TSC. We demonstrate that inhibition of mGluR5 corrects hyperactivity, seizures, and elevated de novo synaptic protein synthesis. Conversely, positive allosteric modulation of mGluR5 results in the exacerbation of hyperactivity and epileptic phenotypes. The data suggest a meaningful therapeutic potential for mGluR5 NAMs in TSC, which warrants clinical exploration and the continued development of mGluR5 therapies.
\end{abstract}

Neuropsychopharmacology (2018) 43, I457-|465; doi:10.1038/npp.2017.295; published online 10 January 2018

\section{INTRODUCTION}

Tuberous sclerosis complex (TSC) is a neurodevelopmental disorder caused by loss of function mutations in either the TSC1 or the TSC2 genes. The gene products integrate cellular cues such as nutritive status to negatively regulate the activity of the mechanistic target of rapamycin complex 1 (mTORC1), a conserved Ser/Thr kinase that organizes organelle biogenesis and maintenance, cell metabolism, proliferation, differentiation, transcription, autophagy, and protein synthesis (Laplante and Sabatini, 2012; Lipton and Sahin, 2014). Individuals with TSC and corresponding animal models of the disease present with cognitive and attention deficits, hyperactivity, epilepsy, and autism spectrum disorder (de Vries et al, 2015). Over a dozen mouse

*Correspondence: Dr M Sahin, Department of Neurology, Boston Children's Hospital, F.M. Kirby Neurobiology Center, Harvard Medical School, 300 Longwood Avenue, Boston, MA 021 I5, USA, Tel: +617919-6258, E-mail: mustafa.sahin@childrens.harvard.edu

${ }^{9}$ These authors contributed equally to this work.

Received 2 August 2017; revised 3 November 2017; accepted 28 November 2017; accepted article preview online 5 December 2017 models of TSC have been generated, exhibiting neurological and developmental deficits (Davis et al, 2015; Ehninger et al, 2008; Tsai et al, 2012). mTOR inhibitors have been investigated in treating somatic and neuropsychiatric manifestations of TSC, but these types of drugs do not address the entire spectrum of the TSC symptomology and have significant adverse effects. Thus, the need for novel therapies addressing the neuropsychiatric symptoms of TSC remains pressing.

Both positive and negative allosteric modulation of the group 1 metabotropic glutamate receptor 5 (mGluR5) have been proposed as a therapeutic intervention in neurodevelopmental disorders. Negative allosteric modulators (NAMs) of mGluR5 have been shown to alleviate long-term memory deficits, excessive repetitive behaviors, motor stereotypies, and social interaction abnormalities in various mouse models of autism (Seese et al, 2014; Silverman et al, 2012; Tian et al, 2015). In addition, mGluR5 NAMs have anticonvulsant and robust anxiolytic properties (Mares et al, 2010; Spooren and Gasparini, 2004). In experiments using slice preparations from $T s c 2^{+/-}$mice, the prototypical mGluR5 NAM, MPEP, reduced ictal bursting and 
ameliorated aberrant plasticity (Potter et al, 2013). In contrast, the mGluR5 positive allosteric modulator (PAM), CDPPB, was reported to ameliorate deficits in learning and memory and chemically induced hippocampal long-term depression in $T s c 2^{+/-}$mice (Auerbach et al, 2011). It is unclear how both inhibition and enhancement of mGluR5 signaling can improve the synaptic and neurological deficits in the $T s c 2^{+/-}$mice. Of note, $T s c 2^{+/-}$mice have a very mild behavioral phenotype and do not develop epilepsy, which occur in individuals with TSC with a prevalence of $>90 \%$. Thus, we took an unbiased approach and tested the effects of mGluR5 negative and positive modulation on core behavioral and epileptic phenotypes in an alternate Tsc2 mutant mouse model that displays spontaneous seizures yet is robust enough to enable chronic in vivo electrographic seizure quantification (Yuan et al, 2012). As activation of the mTOR pathway via inactivation of the Tsc genes is classically associated with protein synthesis in TSC (Dennis et al, 1999; Hoeffer and Klann, 2010; Nie et al, 2015), we also assessed mGluR5 inhibition on synaptic protein synthesis rates.

The Tsc2 mutant mouse model used in the present study contains a null $(\mathrm{K})$ and conditional hypomorphic (C) Tsc2 allele (Pollizzi et al, 2009), expressed with the Synapsin $I$-promoter-driven Cre-recombinase transgene $\left(T s c 2^{k / c-d e l 3-}\right.$ SynICre ${ }^{+}$), producing an allelic series of Tsc2 expression. These mutant mice (from now on referred to as $\mathrm{KC}+$ ) express $\sim 7 \%$ of wild-type levels of functional TSC2 protein and display hyperactivity, cognitive inflexibility, social interaction deficits, as well as increased anxiety-related behavior (Yuan et al, 2012). Previously studied TSC models have either failed to reliably reflect these core, translatable symptoms or have inadequate health or life span to study adult behavioral and epileptic phenotypes. The $\mathrm{KC}+$ mutants on the other hand show robust phenotypes and at the same time improved survival, enabling the behavioral and in vivo electrophysiological identification of adult baseline phenotypes of hyperactivity and spontaneous seizures.

\section{MATERIALS AND METHODS}

\section{Animals}

Mouse breeding and genotyping were performed by a separate investigator than the individual responsible for all behavioral, EEG testing and drug administration. All animals were housed in a temperature-controlled vivarium maintained on a $12 \mathrm{~h}$ light-dark cycle. All procedures were conducted with full approval from the Boston Children's Hospital-Animal Care and Use Committee. Mice used in this study were generated from a mixed-strain background. Tsc2 ${ }^{k / c}$ SynIcre+ (TSC2 KC+) mice and littermate controls were bred by crossing $T s c 2{ }^{k / c}$ SynIcre- male mice with female $T s c 2^{c / c}$ SynIcre+ mice. Control groups for each experiment were littermates of the Tsc2 ${ }^{k / c}$ SynIcre+ mutant mice with the genotype $T s c 2^{c / c}$ SynICre-. Both male and female mice were tested in behavioral, EEG, and molecular assays, in approximately equal numbers for each genotype and treatment group. Mice for behavioral testing were separate cohorts from EEG-tested groups.

\section{Primary Cell Cultures}

Dissociated E18.5 Long Evans Rat (Charles River Labs) cortical neurons were suspended in Neurobasal medium, supplemented with B27, L-glutamine, and penicillin/streptomycin, and plated at a density of $8 \times 10^{5} / 10 \mathrm{~cm}$ plastic tissue culture dish.

\section{Drugs and Treatment}

The novel mGluR5 PAM N-tert-butyl-5-((3-fluorophenyl) ethynyl)- $N$-methylpyrimidine-2-carboxamide (RO6807794; Figure 1a), mGluR5 NAM 2-chloro-4-((2,5-dimethyl-1-(4(trifluoromethoxy)phenyl)-1H-imidazol-4-yl) ethynyl) pyridine (CTEP), as well as the mGluR5 tracer $\left[{ }^{3} \mathrm{H}\right]$-ABP688 were synthesized at Hoffman-La Roche AG (Basel, Switzerland). In $\mathrm{Ca}^{2+}$ mobilization assays with recombinant human mGluR5a expressed in HEK293 cells RO6807794 shows potency of $\mathrm{EC}_{50}=20.3 \mathrm{nM}$ (stimulation with $\mathrm{EC}_{20}$ equivalent of L-glutamate) and no detectable agonist-like activity up to a concentration of $10 \mu \mathrm{M}$. In [3H]-MPEP displacement binding experiments on recombinant human mGluR5a (Lindemann et al, 2011), an $\mathrm{IC}_{50}=55.9 \mathrm{nM}$ was recorded. On all other mGlu receptors, RO6807794 showed no detectable activity up to a concentration of $31.6 \mu \mathrm{M}$.

Acute RO6807794 administration was at $0.3 \mathrm{mg} / \mathrm{kg}$ intraperitoneal (i.p.) with $1 \mathrm{~h}$ pre-treatment time before behavioral and EEG studies. Acute NAM treatment involved a dose of $2 \mathrm{mg} / \mathrm{kg}$ p.o. CTEP formulated as microsuspension in vehicle with a $4 \mathrm{~h}$ pre-treatment time. Chronic NAM administration was at $2 \mathrm{mg} / \mathrm{kg}$ p.o. every $48 \mathrm{~h}$ as described previously (Michalon et al, 2012), from 3 weeks of age until experimental testing was completed; this CTEP regimen resulted in an average brain mGluR5 receptor occupancy of $\sim 80 \%$ as confirmed by in vivo binding with a tritiated version of the mGluR5 PET tracer ABP688 (Michalon et al, 2012). CTEP was dosed with $4 \mathrm{~h}$ pre-treatment time before behavioral and EEG studies. Treatment with vehicle $(0.9 \%$ $\mathrm{NaCl}$ and $0.3 \%$ Tween-80) was performed according to the schedule of mGluR5 PAM or mGluR5 NAM treatment in each experiment.

\section{Autoradiography}

Autoradiography was performed on $10 \mathrm{~mm}$ fresh frozen parasagittal sections with $N=4-5$ sections per genotype and tracer. The assay protocol was as described in (Michalon et al, 2014) for $\left[{ }^{3} \mathrm{H}\right]-\mathrm{ABP} 688$, for $\left[{ }^{3} \mathrm{H}\right]-\mathrm{R} 214127$ the buffer was replaced with $50 \mathrm{mM}$ Tris/ $\mathrm{HCl}, 1.2 \mathrm{mM} \mathrm{MgCl}_{2}$, and $2 \mathrm{mM} \mathrm{CaCl}_{2}(\mathrm{pH}=7.4)$, and $\left[{ }^{3} \mathrm{H}\right]-\mathrm{R} 214127$ was used with a specific activity of $25 \mathrm{Ci} / \mathrm{mmol}$ at a final concentration of $1.5 \mathrm{nM}$. Nonspecific binding was recorded in presence of $10 \mathrm{mM}$ of either MPEP (for $\left[{ }^{3} \mathrm{H}\right]-\mathrm{ABP} 688$ ) or BAY36- 7620 (for $\left.\left[{ }^{3} \mathrm{H}\right]-\mathrm{R} 214127\right)$.

\section{Behavioral Assays}

All behavioral experiments were conducted in the Neurodevelopmental Behavioral Core at Boston Children's Hospital. Behavioral testing was initiated at 6 weeks of age and completed by 8.5 weeks of age. Tests were performed in the 
a Chronic NAM Treatment

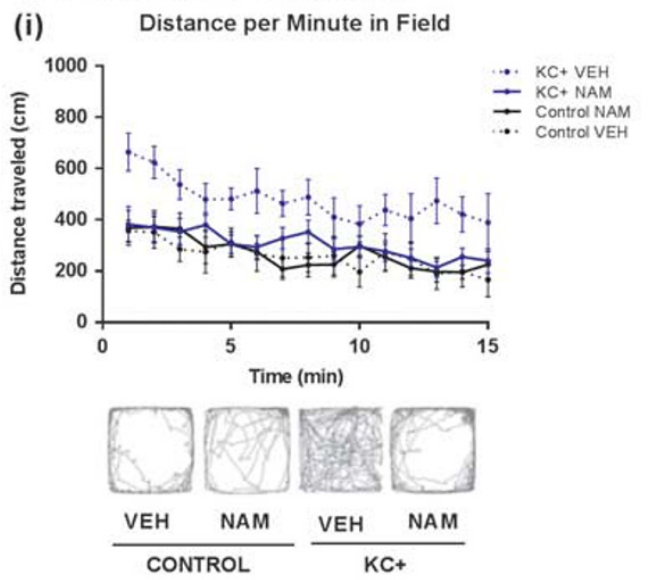

\section{b Representative EEG Traces}

(i)

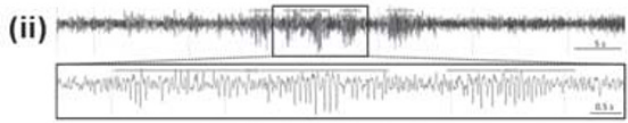

(iii)

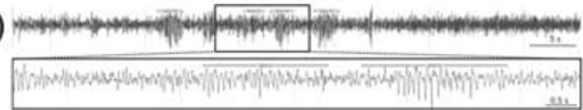

\section{d Acute NAM Treatment}

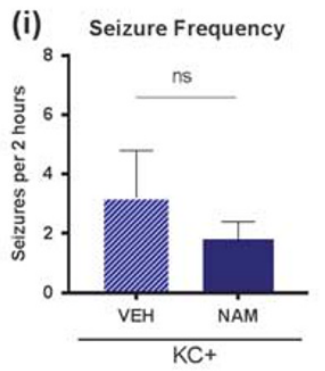

\section{e Chronic NAM Treatment}

(i) Seizure Frequency

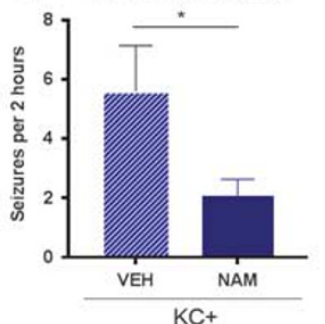

(ii) Frequency to Enter Center Zone

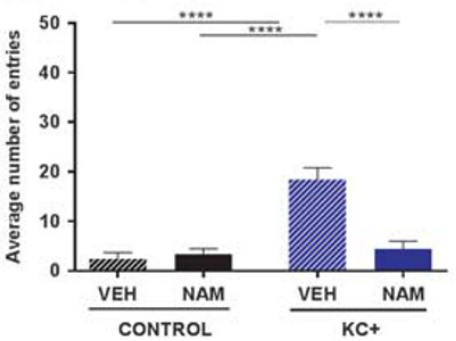

C

Baseline Seizure Differences

Seizure Frequency

Total Seizure Time
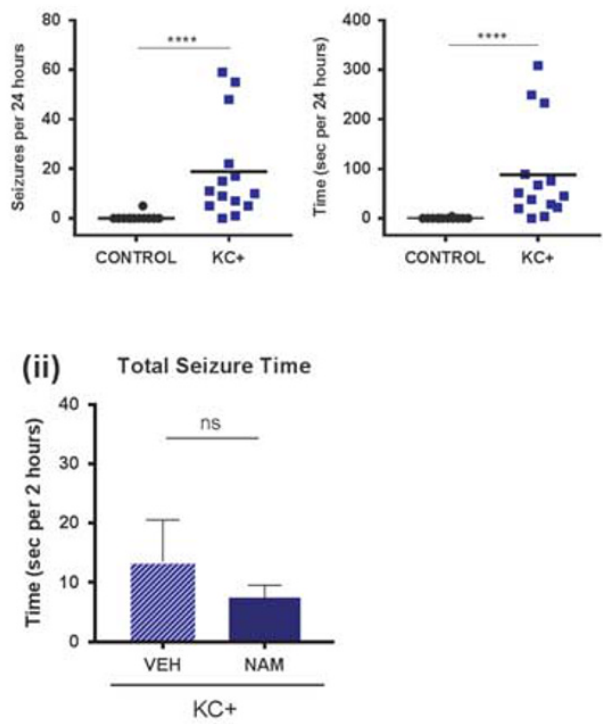

(ii)

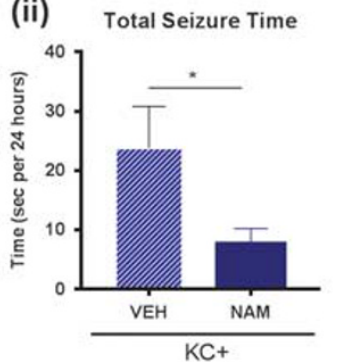

Figure I Negative allosteric modulation of mGluR5 reduces hyperactivity and seizures in Tsc2-deficient mice. (ai) NAM treatment significantly reduces total distance traveled in the open field of $\mathrm{KC}+(\mathrm{N}=14 \mathrm{NAM}$ and $6 \mathrm{VEH})$ but has no effect on control $(\mathrm{N}=9 \mathrm{NAM}$ and $5 \mathrm{VEH})$ mice (two-way repeated measures ANOVA, group $\times$ time, group $F(3,30)=3.744, p=0.02 I$; time $F(I 4,420)=9.60 I, p<0.000 I$ ). (aii) NAM treatment significantly reduces the number of center entries of $\mathrm{KC}+$ mice but has no effect on control mice (two-way ANOVA, treatment $\times$ genotype $F(1,36)=19.19$, $p<0.001$; genotype $F$ $(I, 36)=25.02, p<0.000 \mathrm{I}$; treatment $F(I, 36)=14.65, p=0.0005)$. (b) Representative EEG traces from wireless telemetry (i) non-epileptic baseline in wildtype controls, (ii) spontaneous seizure activity in mutants, (iii) chronic NAM-treated short electrographic seizures in mutants. (c) Baseline seizure frequency, and total seizure time in $24 \mathrm{~h}$ of recording, is higher in $\mathrm{KC}+$ mice relative to controls (mean marked by horizontal bar; $\mathrm{N}=12$ control and $14 \mathrm{KC}+$; MannWhitney test, $p<0.000 \mathrm{I}$ and $<0.000 \mathrm{I}$, respectively). (d) Acute NAM did not significantly reduce seizure frequency (i) and total seizure time (ii) in mutants, $2 \mathrm{~h}$ post treatment ( $\mathrm{VEH} N=5, \mathrm{NAM} \mathrm{N}=8$; unpaired $t$-test, $p=0.3488$ and $0.33 \mathrm{I}$, respectively). (e) Chronic NAM treatment significantly rescued the seizure phenotype, reducing the mean frequency (i) and total seizure time (ii), in $K C+$ mice (VEH N=5, NAM N=6; $p=0.046$ and 0.044 , respectively). Bars indicate mean \pm SEM. ${ }^{*} p<0.05$, ****** $p<0.000 \mathrm{I}$, ns indicates nonsignificant. 
same order, at identical ages and at the same time point in the circadian cycle for all mice.

Open-field experiments were conducted while the animals were 6 weeks of age. Mice were recorded for $15 \mathrm{~min}$ as they freely moved in the open-field arena, as described (Holmes et al, 2001). The open field $(44 \times 44 \mathrm{~cm})$ is enclosed in a soundproof chamber, in a room illuminated at $\sim 40$ lux. Trials were video recorded and total distance traveled, time spent in the center zone, and frequency to enter the center zone were calculated with Noldus EthoVision XT software. Mice experiencing clinical seizures during the $15 \mathrm{~min}$ trial or while being placed into the open field were excluded from data analysis.

\section{In Vivo Electrophysiology}

EEG telemetry unit implantation. Mice, age 6-7 weeks were implanted with wireless telemetry units (PhysioTel ETA-F10; Data Sciences International, DSI, St. Paul, MN) under appropriate sterile technique. Animals were anesthetized by i.p. injection of $100 \mathrm{mg} / \mathrm{kg}$ ketamine (Putney Vet, Portland, ME) and $10 \mathrm{mg} / \mathrm{kg}$ xylazine (Lloyd, Shenandoah, IA). The transmitter was placed intraperitoneally, and electrodes were threaded subcutaneously to the cranium. After skull exposure, hemostasis, and identification of cranial sutures, bregma, and lambda, two burr holes, $1 \mathrm{~mm}$ diameter, were drilled over the right olfactory bulb (reference) and left occipital cortex (active). The telemetry units' epidural electrodes connected to the leads of the transmitter were placed into the burr holes and secured by stainless steel skull screws $(1.1 \mathrm{~mm}$ diameter; PN B002SG89QQ; Antrin Miniature Specialties, Fallbrook, CA). Once in place, the skull screws were covered with dental cement (Dentsply International, Milford, DE). Mice were subcutaneously injected at 0 and $24 \mathrm{~h}$ postoperatively with $5 \mathrm{mg} / \mathrm{kg}$ meloxicam (Norbrook Laboratories, Newry, Northern Ireland) for analgesia. After 1 week of recovery, mice were individually housed in their home cages in a $12 \mathrm{~h}$ light/12 h dark, temperature-, and humidity-controlled chamber with ad libitum access to food and water in preparation for recording.

Video EEG recording and analysis. One-channel video EEG was recorded differentially between the reference and active electrodes. EEG was sampled at $1000 \mathrm{~Hz}$. All data were acquired over a period of $48 \mathrm{~h}$ using the Dataquest ART acquisition software (DSI). All video EEG recordings were scored offline for seizures. A seizure was defined as a rhythmic and sustained train of epileptic spikes $\geqslant 4 \mathrm{~s}$ in duration on EEG. Seizures were quantified by automated seizure detection (Neuroscore (DSI)) (Dhamne et al, 2017) wherein individual spike characteristics such as amplitude, duration, frequency, and inter-spike intervals were used to differentiate seizures from interictal spikes or electrical and mechanical artifacts. Pursuant to the automated spike counts, all traces and detected events were also reviewed by blinded visual inspection of the marked EEG segments to minimize false positive and false negative seizure detection errors, which are unavoidable with any automated seizure detection technique. All marked seizures were then verified against the real-time videos and spectral EEG metrics, before classification as electrographic (no behavioral correlate) or clinical/behavioral (accompanied by freezing or convulsion). Per monitoring period, cumulative seizure number and total seizure time (sum of all individual seizure durations) were computed for each mouse.

\section{Protein Synthesis Quantification}

Synaptoneurosome isolation and NAM treatment in vitro. Synaptoneurosomes (SNs) were isolated from mouse brain cortex of P7-P21 control and $\mathrm{KC}+$ mice, as previously described. Intact SNs were removed (1 $\mathrm{ml}$ at Band $5)$, and incubated with NAM $(10 \mu \mathrm{l}, 50 \mathrm{mg} / \mathrm{ml} \mathrm{DMSO})$, or vehicle for $30 \mathrm{~min}$ at $37^{\circ} \mathrm{C}$, and then placed on ice.

Metabolic labeling and click chemistry. A volume of $5 \mu \mathrm{l}$ of $50 \mathrm{mM}$ L-azidohomoalaine (Click Chemistry Tools) was added to each $\mathrm{SN}$ fraction (to reach a final concentration of $250 \mu \mathrm{M}$ each), and then incubated for $1 \mathrm{~h}$ at $37^{\circ} \mathrm{C}$. SN and cortical fractions were normalized by Bradford assay. THPTA ligand (100 mM, Click Chemistry Tools), CuSO4 (20 mM, Click Chemistry Tools), sodium ascorbate (300 mM, Sigma), and Biotin-PEG3-Azide (20 mM, Click Chemistry Tools) were added to each SN fraction. This reaction was vortexed and then protected from the light for $30 \mathrm{~min}$ at room temperature. Detection of biotinylated proteins was performed by adding High Capacity Streptavidin-Agarose beads (Click Chemistry Tools), washed in PBS, to the biotinylated SN fractions. These samples were incubated overnight at $4{ }^{\circ} \mathrm{C}$ on a rotating wheel. The fractions were then centrifuged at 1000 r.p.m. for $5 \mathrm{~min}$ at $4{ }^{\circ} \mathrm{C}$, and washed three times with $30 \mathrm{mM}$ HEPES, centrifuging after each wash, and discarding the supernatant. The beads were resuspended in $1 \times$ Laemmli buffer and heated at $95^{\circ} \mathrm{C}$ for $5 \mathrm{~min}$. After, the beads and the buffer were centrifuged at 1000 r.p.m. at room temperature for $30 \mathrm{~s}$, and the supernatant was saved and placed on ice.

Pull-down and immunoblotting. SN proteins were separated on a $4-20 \%$ Precision gel and transferred electrophoretically onto Immobilon P membranes. We applied a mouse anti-biotin-HRP antibody (1:2000, Cell Signaling, \#7075) in milk to the membranes, overnight with constant agitation. Proteins were detected in femtogram range, and the signal was quantified by ImageQuant software and normalized to total protein in the same lane.

Puromycin labeling of de novo protein synthesis in cortical neurons. Dissociated E18.5 Long Evans Rat (Charles River Labs) cortical neurons were suspended in Neurobasal medium, supplemented with B27, L-glutamine, and penicillin/streptomycin, and plated at a density of $8 \times 10^{5} / 10 \mathrm{~cm}$ plastic tissue culture dish. At 2 days in vitro, neurons were infected with lentiviral vectors in the presence of polybrene for the knockdown of Tsc2, and maintained at $37^{\circ} \mathrm{C}$ and $5 \%$ $\mathrm{CO}_{2}$ for 14 days. We used an shRNA for $T s c 2$, which we have previously validated (Nie et al, 2015). After this period, neurons were presented with either DMSO or NAM (11 nM) for $24 \mathrm{~h}$ in Neurobasal medium, and then labeled with cellpermeable puromycin $(1 \mu \mathrm{M})$ for $120 \mathrm{~min}$ in fresh Neurobasal medium. Subsequently, the medium was aspirated, and cells were washed in cold PBS $\times 2$. Cells were collected in 


\section{a Acute PAM Treatment}

(i)

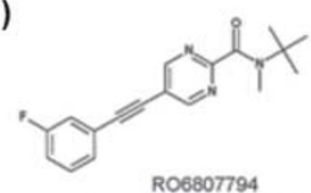

(ii) Distance per Minute in Field

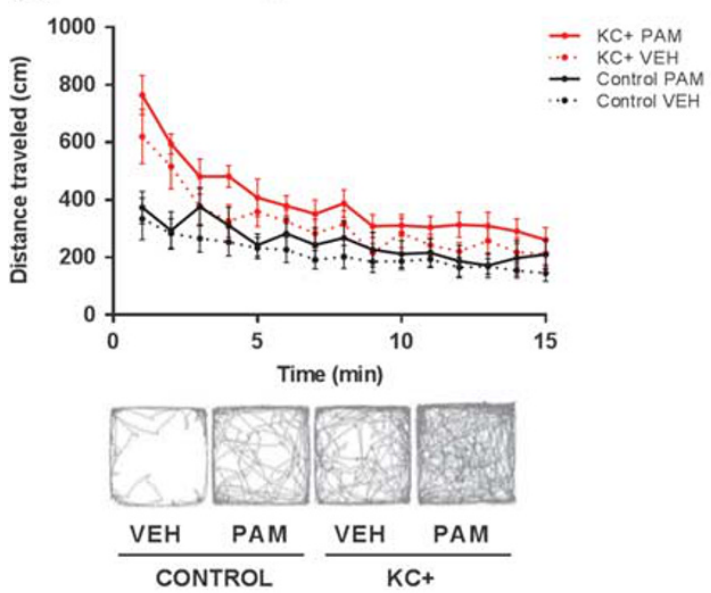

(iii) Frequency to Enter Center Zone

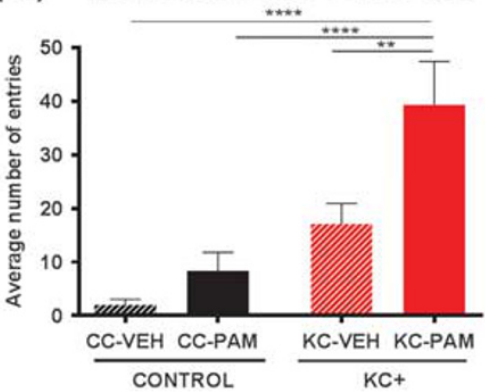

b Representative EEG Traces
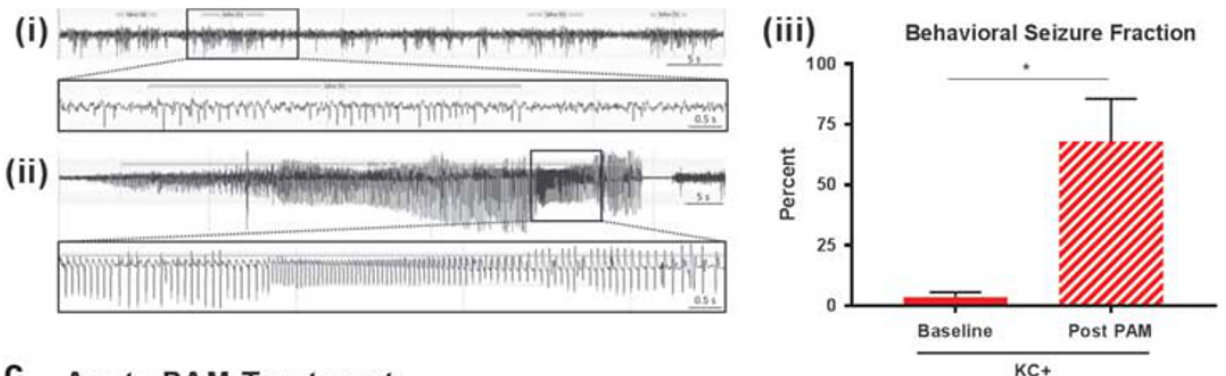

\section{Acute PAM Treatment}

(i)
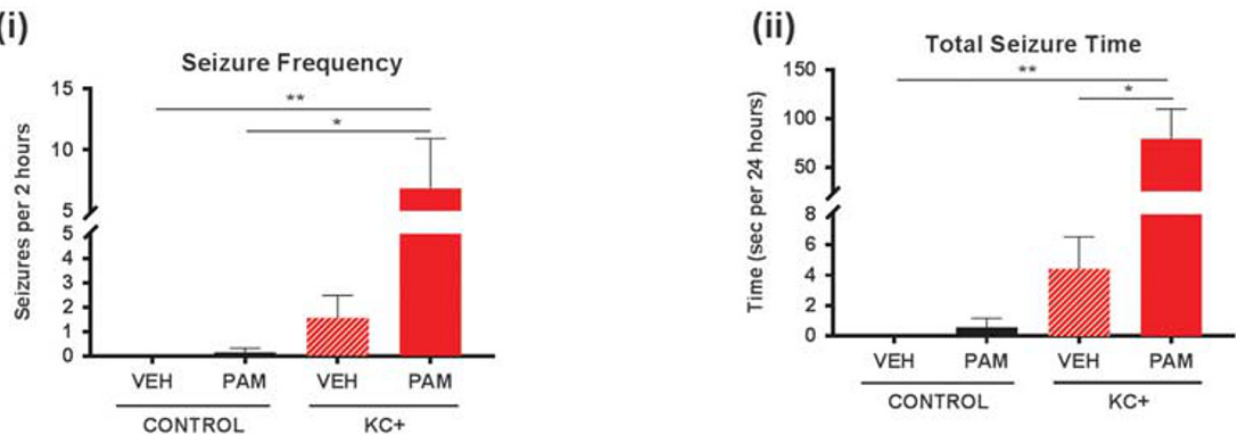

Figure 2 Positive allosteric modulation of mGluR5 exacerbates hyperactivity and seizures in Tsc2-deficient mice. (ai) Chemical structure and composition of the mGluR5 PAM RO6807794. (aii) Tsc2-deficient mice (KC+; N=8 PAM and 9 VEH) travel significantly greater total distances than control mice ( $N=9$ PAM and $13 \mathrm{VEH}$ ) in the open-field assay and PAM treatment significantly increases that distance in $\mathrm{KC}+$ but not control mice (two-way repeated measures ANOVA, group $\times$ time $F(42,490)=2.726, p<0.000$ I; group $F(3,35)=3.908, p=0.017$; time $F(\mid 4,490)=37.26$, $p<0.000 I)$. Subplot indicates pattern of locomotor activity. (aiii) KC+ enter the center zone of the open field significantly more frequently than control mice, and PAM treatment significantly increases the number of entries in the KC+ but not control mice (two-way ANOVA, genotype $\times$ treatment, genotype $F(I, 4 I)=29.45, p<0.000 I ;$ treatment $F(I, 4 \mid)=\mid 1.36, p=0.002$ ). (b) Representative EEG traces from wireless telemetry (i) spontaneous seizure activity in mutants, (ii) acute PAM treatment led to a long clinical seizure in mutants. (iii) PAM administration resulted in a significant increase in the fraction of clinical seizures, from 3.6\% at baseline to $68 \%$ post treatment $(N=7 \mathrm{KC}+\mathrm{PAM}$, paired $t$-test, $p=0.02$ ). (c) Acute PAM administration significantly exacerbated both seizure frequency (Kruskal-Wallis ANOVA, $p=0.002 \mathrm{I}$ ) and total seizure time (Kruskal-Wallis ANOVA, $p=0.0004)$ in KC+ mice in $2 \mathrm{~h}$ following treatment. PAM-treated KC+ had significantly more seizures $(p=0.0 \mathrm{I})$ and significantly longer seizures $(p=0.003)$ than PAM-treated control mice, as compared by Dunn's post hoc tests. PAM-treated KC+ animals also had significantly more seizures than VEH-treated $K C+$ animals $(p=0.04)$. There was no effect of PAM treatment on control animals $(N=6$ control VEH, 6 control PAM, $7 \mathrm{KC}+\mathrm{VEH}$, and $7 \mathrm{KC}+\mathrm{PAM})$. Data are plotted as mean \pm SEM. $* p<0.05, * * p<0.01, * * * * p<0.0001$. 
$3 \mathrm{ml}$ of PBS, and spun at $500 \mathrm{~g}$ for $5 \mathrm{~min}$ at $4{ }^{\circ} \mathrm{C}$. PBS was then aspirated, and cells were lysed in $100 \mu \mathrm{L}$ of RIPA buffer (30 mM HEPES, $150 \mathrm{mM} \mathrm{NaCl}, 1 \% \mathrm{NP}-40,0.5 \mathrm{~g}$ sodium dexoycholate). A volume of $20 \mathrm{ml}$ of RIPA was supplemented with one tablet of EDTA-free Complete protease inhibitor and one tablet of PhosStop (Roche), and kept on ice for $5 \mathrm{~min}$. Samples were spun at $16 \mathrm{~g}$ for $10 \mathrm{~min}$ at $4{ }^{\circ} \mathrm{C}$, and supernatant was used for protein detection. Puromycinlabeled proteins were separated on a 4-20\% Precision gel and transferred electrophoretically onto Immobilon $\mathrm{P}$ membranes. Monoclonal anti-puromycin antibody (Kerafast 1:1000, \#EQ0001) was detected and measured by densitometry.

Quantification and statistical analysis. All statistical analyses were performed using GraphPad Prism (v 5.01; GraphPad Software, La Jolla, CA), with significance level defined as $p<0.05$. On the basis of normality of data (determined by Shapiro-Wilk test) either parametric or nonparametric tests were used. For normally distributed data, differences between groups for behavioral testing were analyzed with a one-way ANOVA or two-way ANOVA, followed by a Tukey post-test between all groups. Unpaired $t$-tests and one-way ANOVA (followed by a Tukey post-test) were used to compare frequency of seizures and total seizure time when comparing two or three groups, respectively. Non-normally distributed data were analyzed in a parallel fashion using the Mann-Whitney $U$-test and Kruskal-Wallis ANOVA (followed by Dunn's post-test). Paired $t$-test was used to compare behavioral seizure fractions before and after PAM treatment. Unpaired $t$-tests and one-way ANOVA (followed by a Tukey post-test) were used to compare treatment groups for in vivo and in vitro biochemical assays when comparing two or three groups, respectively. Repeated measures analysis of variance was used to compare genotypes for most of the behavioral assays. Test groups were unblinded after analysis was complete.

\section{RESULTS}

We first examined the effect of the mGluR5 NAM CTEP (Lindemann et al, 2011) on the $\mathrm{KC}+$ mice and control littermates. Chronic NAM was administered at $2 \mathrm{mg} / \mathrm{kg}$ p.o. every $48 \mathrm{~h}$ as described previously (Michalon et al, 2012), from 3 weeks of age until behavior or EEG testing was complete ( 8 or 7 weeks of age, respectively). Treatment of $\mathrm{KC}+$ mice with NAM significantly decreased hyperactive behaviors to levels comparable with vehicle-treated control mice, demonstrated by total distance traveled and time spent in the center of the arena (Figure 1ai-ii).

We also performed intracranial EEG recordings by wireless telemetry accompanied by video tracking over a $24 \mathrm{~h}$ period to monitor electrographic and clinical seizures (Figure 1b). Untreated $\mathrm{KC}+$ mutants experienced regularly occurring unprovoked electrographic and clinical seizures that are absent in littermate controls (Figure 1bi and ii and c; Supplementary Video 1). To specifically probe the impact of modulating mGluR5 signaling on seizure activity of the mutants, we compared acute and chronic mGluR5 NAM treatment paradigms. Acute treatment with the mGluR5 NAM resulted in reduced seizure frequency and reduced total seizure time in $\mathrm{KC}+$ mice, which, however, were not statistically significant (Figure 1d). Chronic NAM treatment, in contrast, resulted in a significant reduction of seizure frequency and total seizure time in $\mathrm{KC}+$ mice, by $65 \%$ relative to vehicle treatment (Figure 1 biii and e), but seizure duration was unchanged.

We then asked whether the potent and selective mGluR5 PAM, RO6807794, (Figure 2ai, also see Materials and Methods) would impact the behavioral phenotypes of $\mathrm{KC}+$ mice. In an open-field assay, single-dose treatment with the mGluR5 PAM drastically increased baseline hyperactivity phenotypes in mutants, which entered the center zone with significantly greater frequency and duration than vehicletreated controls (Figure 2aii and iii). In control animals, the mGluR5 PAM had a modest and not statistically significant effect on locomotor activity and center crossings (Figure 2aii and iii).

Acute mGluR5 PAM administration also drastically exacerbated the epileptic phenotype in mutant mice relative to pre-injection baseline (Figure 2bi and ii), while the same PAM dose did not trigger any measureable seizure activity in control mice. Two hours following PAM treatment, KC+ mice demonstrated significant increase in seizure frequency and total seizure time for the $2 \mathrm{~h}$ monitoring period, while PAM-treated control mice showed no seizure activity (Figure 2c). The fraction of clinical (as opposed to strictly electrographic) seizures also significantly increased with mGluR5 PAM administration in $\mathrm{KC}+$ mice (Figure 2biii; Supplementary Video 2).

One hypothesis for the efficacy of mGluR5 NAM in the $\mathrm{KC}+$ mice may be upregulation of mGluR 5 receptors in the brain. In fact, a previous study using $T s c 2^{+/-}$mice reported that there was a small, but significant, increase in mGluR5 protein expression in adult $T s c 2^{+/-}$mice compared to their wild-type littermates (Potter et al, 2013). We measured the abundance of mGluR5 by means of autoradiography using the mGluR5-selective radiotracer $\left[{ }^{3} \mathrm{H}\right]-\mathrm{ABP} 688$, which is being used in 11C- or 18F-radiolabeled version as human mGluR5 PET tracer (Ametamey et al, 2006; Hintermann et al, 2007; Treyer et al, 2008). However, the data indicate a consistent reduction of mGluR5 abundance in $\mathrm{KC}+$ mice compared to control littermates in cortex $(-10.7 \%)$, hippocampus $(-19.8 \%)$, and striatum (both $-20.1 \%$ ), as well as alterations of mGluR1 abundance, as measured by $\left[{ }^{3} \mathrm{H}\right]$ R214127 binding, in cortex $(+15 \%)$, hippocampus (+7.6\%), striatum $(-11.2 \%)$, and cerebellum $(-19.9 \%)$ (Figure 3a). Given the modest changes in mGluR5 expression levels, it is unlikely that either the basic phenotype of the $\mathrm{KC}+$ mice or the drug effects are mediated by changes in the expression levels of the drug target.

To determine whether behavioral and neurophysiological effects of mGluR5 inhibition are linked to core molecular pathophysiology of TSC, we examined whether the NAM would normalize the elevated synaptic protein synthesis rate in these mice. Given that much of the pathology of mTORC1 hyperactivation, as occurs with Tsc2 mutation, is associated with increased transcription, ribosomal biogenesis and translation (Dennis et al, 1999; Hoeffer and Klann, 2010; Nie et al, 2015), we hypothesized that inhibiting mGluR5 would reduce the level of synaptic protein synthesis. While this contradicts the finding of decreased protein synthesis specifically within the hippocampal slices of $\mathrm{Tsc}^{+}-$mice 
a mGluR5 Autoradiography

(i)

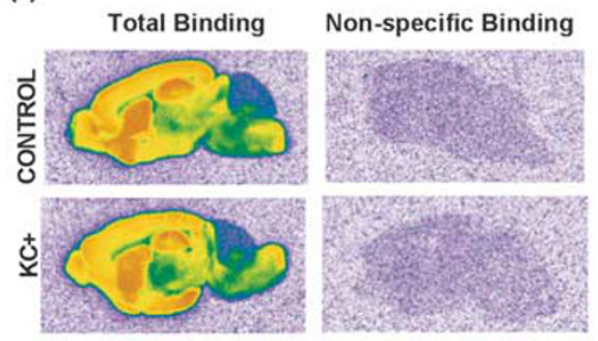

(ii)

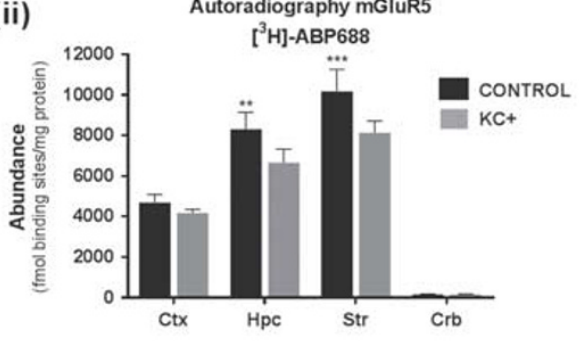

b

(i)

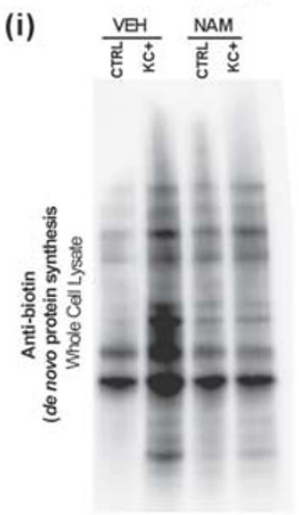

(ii) Anti-biotin de novo protein synthesis

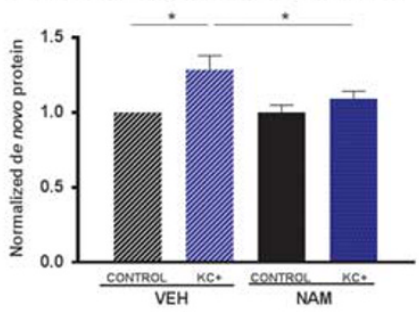

C SUnSET Assay

(i)
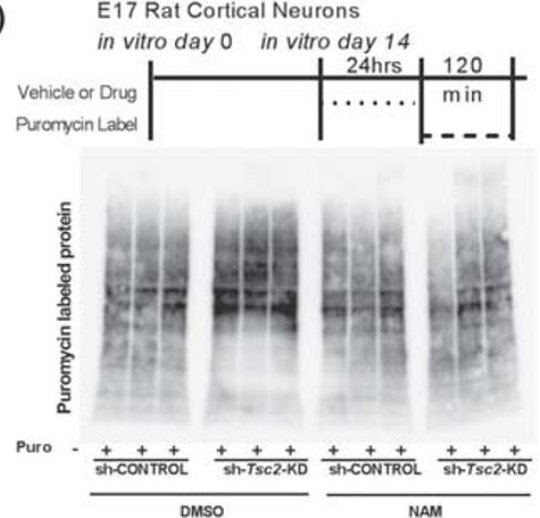

(ii) Puromycin de novo protein synthesis

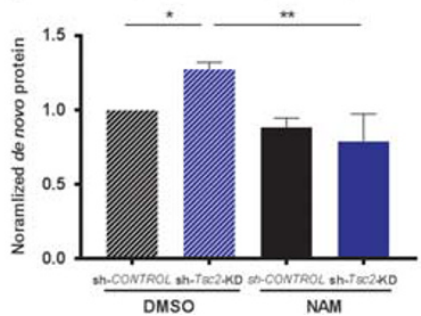

Figure 3 Negative allosteric modulation of mGluR5 reverses aberrant increase in synaptic and global neuronal protein synthesis in Tsc2 mutant brains and cells. (ai) Abundance of mGluR5 quantified by means of autoradiography using the mGluR5-selective tracers $\left[{ }^{3} \mathrm{H}\right]$-ABP688. (aii) The data indicate a consistent reduction of mGluR5 abundance in TSC2-KD compared to WT mice in cortex $(-10.7 \%)$, hippocampus $(-19.8 \% ; p<0.0046)$ and striatum (both $-20.1 \%$; $p<0.0003$ ). (bi) BONCAT assay of metabolically labeled de novo synthesized proteins from KC+ and control cortical SNs ( $N=3$ per condition). (bii) Antibiotin-labeled blot demonstrated a significant increase in total de novo protein level in $\mathrm{KC}+$ relative to control samples after vehicle (VEH) treatment that was normalized by NAM treatment (one-way ANOVA, $p=0.0117$ and 0.0353). (ci) In vitro SUnSET puromycin labeling experimental design, and blot of puromycin-labeled proteins and Coomassie Blue staining. (cii) Increased de novo protein synthesis in sh-Tsc2-KD cells, compared to control cells ( $N=3$ for each condition, one-way ANOVA, control vs Tsc2 KD p =0.0475, VEH Tsc2 KD vs NAM Tsc2 KD p =0.00 I 7). NAM treatment significantly reduced protein synthesis in mutants $(p=0.0017)$. Data are plotted as normalized mean \pm SEM. ${ }^{*} p<0.05, * * * 0.01, * * * p<0.001$.

seen by Auerbach et al, 2011, it is consistent with the hypothesis that mTOR is a mediator of the mGluR5dependent enhanced protein synthesis in Fragile $\mathrm{X}$ syndrome. Isolated cortical $\mathrm{SN}$ fractions from $\mathrm{KC}+$ mutants were treated with NAM in vitro. Protein synthesis was quantified using bio-orthogonal non-canonical amino-acid tagging, in which SN preparations were labeled with the methionine analog L-azidohomoalanine (L-AHA) followed by $\mathrm{Cu}^{2+}$-mediated cycloaddition of biotin and pull down with Neutravidin-coated beads to isolate and quantify L-AHA-labeled de novo-synthesized proteins. An essentially complete correction of the elevated protein synthesis rate was observed in mGluR5 NAM-treated $\mathrm{KC}+\mathrm{SNs}$, compared to vehicle-treated mutant fractions (Figure $3 \mathrm{~b}$ ). These findings were corroborated with a different approach, using cultured WT rat cortical neurons in vitro in which TSC2 expression was reduced by shRNA Tsc2-knockdown (KD). De novo protein synthesis as assessed by puromycin labeling (SUnSET assays) (Schmidt et al, 2009) was increased in the Tsc2$\mathrm{KD}$ neurons compared to controls and was reduced to control levels by treatment with the mGluR5 NAM (Figure 3c). Taken together these data indicate that mGluR5 NAM treatment can normalize excessive protein synthesis in Tsc2-deficient neurons in vivo and in vitro.

\section{DISCUSSION}

Our findings demonstrate that mGluR5 inhibition has therapeutic potential for the treatment of TSC as it corrects core phenotypes of the disease including seizures and hyperactivity, as well as elevated synaptic protein synthesis. The exacerbation of seizures and hyperactivity in the KC+ model after treatment with the mGluR5 PAM RO6807794 corroborate our findings with the mGluR5 NAM CTEP.

The mGluR5 PAM-mediated exacerbation of seizures and hyperactivity in the $\mathrm{KC}+$ model was unexpected given the good tolerability of the mGluR5 PAM in control animals in the current studies, and in light of previous studies in $T s c 2^{+/-}$ mice with the PAM CDPPB (Auerbach et al, 2011) in which no adverse effects were reported. The apparent differences in tolerability could be due to the fact that there are no spontaneous seizures and no proconvulsive activity in $\mathrm{Tsc}^{+/-}$mice, which renders them less vulnerable to drugs enhancing excitatory neurotransmission such as mGluR5 PAMs compared to $\mathrm{KC}+$ mice. Recent studies have revealed that the tolerability of mGluR5 PAMs in wild-type animals depends on their shift-factor, a compound-intrinsic property, which reflects the potentiation of the activity of the agonist on the receptor in presence of the PAM (the shiftfactor means by how many fold the $\mathrm{EC}_{50}$ of the agonist is potentiated at saturating concentrations of the PAM) 
(Parmentier-Batteur et al, 2014). These studies suggest that in wild-type animals mGluR5 PAMs with a shift factor up to about twofold, such as RO6807794, are well tolerated, while PAMs with higher shift-factors can cause seizures and neurotoxic lesions, depending on the dose used. The shift factor of CDPPB is three- to ninefold (Kinney et al, 2005) and the dose used by Auerbach et al (2011) was $10 \mathrm{mg} / \mathrm{kg}$ i.p. Previous studies revealed that doses of 30 and $100 \mathrm{mg} / \mathrm{kg}$ CDPPB p.o. in rat achieved brain receptor occupancy of 14 and $5 \%$, which is conceivable given the $<1 \%$ free fraction (Gilmour et al, 2013) of the compound. Therefore, it is likely that the receptor occupancy reached in the study by Auerbach et al was $<10 \%$, which is probably insufficient to trigger seizures or other adverse events upon acute dosing in $T s c 2^{+/-}$or wild-type animals despite the compound's high intrinsic shift factor. Thus, the presence of PAM induced seizures in our $\mathrm{KC}+$ model but their absence in the $\mathrm{Tsc}^{+/-}$model is likely due to both the differences in seizure susceptibility between the models and the low levels of receptor occupancy in the Auerbach study.

Our finding that chronic mGluR5 NAM treatment improved the hyperactivity and seizure phenotype in the $\mathrm{KC}+$ model is consistent with the observations that were previously reported in the $T s c 2^{+/-}$mice, with some important differences (Potter et al, 2013). First, we do not detect any increase in mGluR5 receptor expression in our mouse model unlike Potter and colleagues reported for the $\mathrm{Tsc}^{+/-}$mice. Second, instead of acute treatment with a short-acting mGluR5 NAM (MPEP: half-life in mice is ca. 20 min (Lindemann et al, 2011)), we used chronic treatment with a long-acting compound, similar to what would be expected in patients. We observed significant and sustained improvement in $\mathrm{KC}+$ mice in terms of hyperactivity and seizures. These data are consistent with the concept that the loss of the TSC protein complex leads to increased mTORC1 activity, which causes enhanced mGluR5 downstream signaling and increasing protein synthesis, contributing to the abnormal behavioral and seizure phenotypes seen in these mice. The mechanism(s) by which reduction in mGluR5 signaling ameliorates the mTOR hyperactivity and excessive protein synthesis in Tsc2-deficient neurons are not fully resolved. Future experiments using the $\mathrm{KC}+$ mice can shed light on the different steps in signaling downstream of mGluR5 receptors in the context of neuronal TSC1/2 deficiency.

\section{FUNDING AND DISCLOSURE}

L.L., M.H., and G.J. are employees of Roche. M.S. has received research funding from Roche, Novartis, Pfizer and LAM Therapeutics on projects unrelated to work presented here. M.S. has served on the Scientific Advisory Board of SAGE Therapeutics. A.R. has received funding from Brainsway, CRE Medical, Eisai, Neuropace, SAGE Therapeutics, Roche, and Takeda, serves on the advisory board of NeuroRex, and is a co-founder and advisor to Neuromotion-none of the above relate to the present project. D.J.K. has been a consultant to Astra-Zeneca and Eisai. The novel mGluR5 PAM N-tert-butyl-5-((3fluorophenyl)ethynyl)- $N$-methylpyrimidine-2-carboxamide (RO680794) was developed and synthesized at Hoffman-La
Roche AG (Basel, Switzerland). The rest of the authors declare no conflicts of interest.

\section{ACKNOWLEDGMENTS}

We are indebted to members of the Sahin lab for critical reading of the manuscript.

This study was supported by the $\mathrm{BCH}$ Neurodevelopmental Behavioral Core of the Intellectual and Developmental Disabilities Research Center (NIH U54 HD090255), the $\mathrm{BCH}$ Translational Neuroscience Center, and the Experimental Neurophysiology Core, Nancy Lurie Marks Family Foundation, and the Boston Children's Hospital Translational Research Program (to M.S. and A.R.).

\section{AUTHOR CONTRIBUTIONS}

Conceived and designed the experiments: A.R., L.L., and M.S.; performed the experiments: E.K., S.M.S., S.C.D., J.O.L., C.E.S., S.H.T.L., and M.H.; analyzed the data: S.C.D., J.O.L., M.E.M., A.R., M.H., and M.S.; contributed reagents/materials/ analysis tools: D.J.K., L.L., G.J., J.L.S., and J.R.D.; drafted the article: E.K., S.M.S., S.C.D., L.L., M.E.M., and M.S.; revised the article: E.K., S.M.S., S.C.D., J.O.L., L.L., C.E.S., S.H.T., M.E.M., J.L.S., J.R.D, D.J.K., A.R., and M.S.

\section{REFERENCES}

Ametamey SM, Kessler LJ, Honer M, Wyss MT, Buck A, Hintermann S et al (2006). Radiosynthesis and preclinical evaluation of $11 \mathrm{C}-\mathrm{ABP} 688$ as a probe for imaging the metabotropic glutamate receptor subtype 5. J Nucl Med 47: 698-705.

Auerbach BD, Osterweil EK, Bear MF (2011). Mutations causing syndromic autism define an axis of synaptic pathophysiology. Nature 480: 63-68.

Davis PE, Peters JM, Krueger DA, Sahin M (2015). Tuberous sclerosis: a new frontier in targeted treatment of autism. Neurotherapeutics 12: 572-583.

de Vries PJ, Whittemore VH, Leclezio L, Byars AW, Dunn D, Ess $\mathrm{KC}$ et al (2015). Tuberous sclerosis associated neuropsychiatric disorders (TAND) and the TAND Checklist. Pediatr Neurol 52: 25-35.

Dennis PB, Fumagalli S, Thomas G (1999). Target of rapamycin (TOR): balancing the opposing forces of protein synthesis and degradation. Curr Opin Genet Dev 9: 49-54.

Dhamne SC, Silverman JL, Super CE, Lammers SHT, Hameed MQ, Modi ME et al (2017). Replicable in vivo physiological and behavioral phenotypes of the Shank3B null mutant mouse model of autism. Mol Autism 8: 26.

Ehninger D, Han S, Shilyansky C, Zhou Y, Li W, Kwiatkowski DJ et al (2008). Reversal of learning deficits in a Tsc2+/- mouse model of tuberous sclerosis. Nat Med 14: 843-848.

Gilmour G, Broad LM, Wafford KA, Britton T, Colvin EM, Fivush A et al (2013). In vitro characterisation of the novel positive allosteric modulators of the mGlu(5) receptor, LSN2463359 and LSN2814617, and their effects on sleep architecture and operant responding in the rat. Neuropharmacology 64: 224-239.

Hintermann S, Vranesic I, Allgeier H, Brulisauer A, Hoyer D, Lemaire $M$ et al (2007). ABP688, a novel selective and high affinity ligand for the labeling of mGlu5 receptors: identification, in vitro pharmacology, pharmacokinetic and biodistribution studies. Bioorg Med Chem 15: 903-914.

Hoeffer CA, Klann E (2010). mTOR signaling: at the crossroads of plasticity, memory and disease. Trends Neurosci 33: 67-75. 
Holmes A, Hollon TR, Gleason TC, Liu Z, Dreiling J, Sibley DR et al (2001). Behavioral characterization of dopamine D5 receptor null mutant mice. Behav Neurosci 115: 1129-1144.

Kinney GG, O'Brien JA, Lemaire W, Burno M, Bickel DJ, Clements $\mathrm{MK}$ et al (2005). A novel selective positive allosteric modulator of metabotropic glutamate receptor subtype 5 has in vivo activity and antipsychotic-like effects in rat behavioral models. J Pharmacol Exp Ther 313: 199-206.

Laplante M, Sabatini DM (2012). mTOR signaling in growth control and disease. Cell 149: 274-293.

Lindemann L, Jaeschke G, Michalon A, Vieira E, Honer M, Spooren W et al (2011). CTEP: a novel, potent, long-acting, and orally bioavailable metabotropic glutamate receptor 5 inhibitor. J Pharmacol Exp Ther 339: 474-486.

Lipton JO, Sahin M (2014). The neurology of mTOR. Neuron 84: 275-291.

Mares P, Mikulecka A, Ticha K, Lojkova-Janeckova D, Kubova H (2010). Metabotropic glutamate receptors as a target for anticonvulsant and anxiolytic action in immature rats. Epilepsia $\mathbf{5 1}$ (Suppl 3): 24-26.

Michalon A, Bruns A, Risterucci C, Honer M, Ballard TM, Ozmen L et al (2014). Chronic metabotropic glutamate receptor 5 inhibition corrects local alterations of brain activity and improves cognitive performance in fragile $\mathrm{X}$ mice. Biol Psychiatry 75: 189-197.

Michalon A, Sidorov M, Ballard TM, Ozmen L, Spooren W, Wettstein JG et al (2012). Chronic pharmacological mGlu5 inhibition corrects fragile $X$ in adult mice. Neuron 74: 49-56.

Nie D, Chen Z, Ebrahimi-Fakhari D, Di Nardo A, Julich K, Robson VK et al (2015). The stress-induced Atf3-gelsolin cascade underlies dendritic spine deficits in neuronal models of tuberous sclerosis complex. J Neurosci 35: 10762-10772.

Parmentier-Batteur S, Hutson PH, Menzel K, Uslaner JM, Mattson BA, O'Brien JA et al (2014). Mechanism based neurotoxicity of mGlu5 positive allosteric modulators-development challenges for a promising novel antipsychotic target. Neuropharmacology 82: 161-173.
Pollizzi K, Malinowska-Kolodziej I, Doughty C, Betz C, Ma J, Goto J et al (2009). A hypomorphic allele of Tsc2 highlights the role of TSC1/TSC2 in signaling to AKT and models mild human TSC2 alleles. Hum Mol Genet 18: 2378-2387.

Potter WB, Basu T, O'Riordan KJ, Kirchner A, Rutecki P, Burger C et al (2013). Reduced juvenile long-term depression in tuberous sclerosis complex is mitigated in adults by compensatory recruitment of mGluR5 and Erk signaling. PLoS Biol 11: e1001627.

Schmidt EK, Clavarino G, Ceppi M, Pierre P (2009). SUnSET, a nonradioactive method to monitor protein synthesis. Nat Methods 6: 275-277.

Seese RR, Maske AR, Lynch G, Gall CM (2014). Long-term memory deficits are associated with elevated synaptic ERK1/2 activation and reversed by mGluR5 antagonism in an animal model of autism. Neuropsychopharmacology 39: 1664-1673.

Silverman JL, Smith DG, Rizzo SJ, Karras MN, Turner SM, Tolu SS et al (2012). Negative allosteric modulation of the mGluR5 receptor reduces repetitive behaviors and rescues social deficits in mouse models of autism. Sci Transl Med 4: 131ra151.

Spooren W, Gasparini F (2004). mGlu5 receptor antagonists: a novel class of anxiolytics? Drug News Perspect 17: 251-257.

Tian D, Stoppel LJ, Heynen AJ, Lindemann L, Jaeschke G, Mills AA et al (2015). Contribution of mGluR5 to pathophysiology in a mouse model of human chromosome 16p11.2 microdeletion. Nat Neurosci 18: 182-184.

Treyer V, Streffer J, Ametamey SM, Bettio A, Blauenstein P, Schmidt $\mathrm{M}$ et al (2008). Radiation dosimetry and biodistribution of 11C-ABP688 measured in healthy volunteers. Eur J Nucl Med Mol Imaging 35: 766-770.

Tsai PT, Hull C, Chu Y, Greene-Colozzi E, Sadowski AR, Leech JM et al (2012). Autistic-like behaviour and cerebellar dysfunction in Purkinje cell Tsc1 mutant mice. Nature 488: 647-651.

Yuan E, Tsai PT, Greene-Colozzi E, Sahin M, Kwiatkowski DJ, Malinowska IA (2012). Graded loss of tuberin in an allelic series of brain models of TSC correlates with survival, and biochemical, histological and behavioral features. Hum Mol Genet 21: 4286-4300.

Supplementary Information accompanies the paper on the Neuropsychopharmacology website (http://www.nature.com/npp) 